\title{
Soziale Sicherung für Migranten unter besonderer Berücksichtigung der Unionsbürgerschaft
}

- Eine Untersuchung am Beispiel der Grundsicherung für Arbeitsuchende -

\section{Inklusion als Anliegen existenzsichernder Sozialleistungen}

Mit dem Sozialgesetzbuch sollen gemäß § 1 I SGB I soziale Gerechtigkeit und soziale Sicherheit verwirklicht werden. Darunter sind insbesondere die Sicherung eines menschenwürdigen Daseins und der Schutz der freien Persönlichkeitsentfaltung zu verstehen. Nach $\S 9$ SGB I hat jeder, der nicht in der Lage ist, seinen Lebensunterhalt selbst oder durch die Hilfe anderer zu bestreiten, einen Anspruch auf persönliche und wirtschaftliche Hilfe. Der Gesetzgeber versteht die Sozialhilfe als Ausdruck der sozialen Inklusion, die dem Einzelnen ein menschenwürdiges Leben als Teil der Gesellschaft ermöglichen soll. ${ }^{1}$ Die in $\S 9$ SGB I verankerten Leitlinien treffen keine Unterscheidung nach der Staatsangehörigkeit des Berechtigten. Sie knüpfen an das bloße Mensch-Sein an. Einzige Anspruchsvoraussetzung ist die Hilfebedürftigkeit, also das Unvermögen, seinen Lebensunterhalt aus eigener Kraft zu bestreiten oder sich in besonderen Bedarfslagen zu versorgen.

Aus dem Anliegen der Eingliederung des Hilfebedürftigen in die Gesellschaft ergeben sich jedoch Spielräume: Wer ist der Gesellschaft zugehörig? Welche Ansprüche sind denjenigen zuzugestehen, die keinen eigenen Beitrag zur Finanzierung des Sozialhilfesystems geleistet haben? Die gesetzlichen Regelungen über die Gewährung steuerfinanzierter Sozialleistungen an Migranten geben daher Aufschluss über das Maß an Solidarität, das die Aufnahmegesellschaft zu gewähren bereit ist. Sie sollen am Beispiel der Grundsicherung für Arbeitsuchende nach dem SGB II erläutert werden. Dabei ist zunächst auf die Zulässigkeit der Aufenthaltsbegründung in der Bundesrepublik einzugehen (II.). Sodann sind die speziellen Anspruchsvoraussetzungen (III.) und Ausschlusstatbestände der Grundsicherung für Ausländer zu schildern (IV.). Die Regelungen sind schließlich auf ihre völker- und europarechtliche Tragfähigkeit hin zu untersuchen (V.).

\section{Gewöhnlicher Aufenthalt als Anknüpfungsmoment für die Leistungsberechtigung}

Leistungen der Grundsicherung für Arbeitsuchende erhalten gemäß $§ 7$ I 1 SGB II erwerbsfähige Hilfebedürftige mit gewöhnlichem Aufenthalt in der Bundesrepublik, die das 15. Lebensjahr vollendet und das Renteneintrittsalter noch nicht erreicht haben. Der territoriale Bezug reflektiert das in $\S 30$ SGB I für das gesamte Sozialrecht vorgegebene „Territorialprinzip“ als Anknüpfungsmoment der Leistungsberechtigung.

1 BVerfGE 125, 175. 


\section{Das „,Territorialprinzip “ im Recht der sozialen Sicherheit}

Staats- und völkerrechtlichen Ursprungs, ist das „Territorialprinzip“ Ausdruck und Folge der auf das Staatsgebiet beschränkten Rechtssetzungsbefugnis der Nationalstaaten: keinem Staat ist es gestattet, außerhalb seiner Grenzen Hoheitsgewalt auszuüben. Dies hat nicht zur Folge, dass es Staaten generell verwehrt wäre, Sachverhalte außerhalb des eigenen Territoriums zu regeln. Es bedarf lediglich einer tragfähigen, legitimen Anknüpfung. Sie kann territorialer, aber auch personaler Natur sein. Der Sozialstaat darf daher Leistungen an eigene Staatsbürger erbringen, die sich im Ausland aufhalten. Er kann sich aber auch auf die Regelung von Sachverhalten auf dem eigenen Territorium beschränken. $^{2}$

Der Begriff „Territorialprinzip“ greift in der Leistungsverwaltung jedoch zu kurz, wird hier doch weniger Hoheitsgewalt ausgeübt als einer staatlichen Einstandspflicht nachgekommen. ${ }^{3}$ Diese zielt auf die Gewährung gleicher Lebenschancen, die Sicherung von Solidarität, und soll soziale Gerechtigkeit bewirken. § 30 I SGB I soll also nicht die sozialstaatlichen Befugnisse auf das Territorium der Bundesrepublik begrenzen. Vielmehr wollte der Gesetzgeber mit der Etablierung des Wohnortprinzips von einer Anknüpfung der Leistungsgewährung an die Staatsangehörigkeit absehen. ${ }^{4}$ Durch die Anwendbarkeitserklärung nationalen Sozialrechts auf dem inländischen Territorium werden „Fremde“ in den Verband der Leistungsberechtigten integriert. ${ }^{5}$ Der Sozialstaat ist mithin allen Personen verpflichtet, die ihren Lebensmittelpunkt in der Bundesrepublik haben und ihr dadurch besonders verbunden sind.

\section{Begründung des gewöhnlichen Aufenthalts}

Der gewöhnliche Aufenthalt setzt voraus, dass sich jemand unter Umständen in der Bundesrepublik aufhält, die darauf schließen lassen, dass der Aufenthalt nicht nur vorübergehend ist, § 30 III 2 SGB I. Dies ist der Fall, wenn die Bundesrepublik faktisch und dauerhaft zum Lebensmittelpunkt gemacht wird, wenn also der Aufenthalt nicht nur kurzfristig genommen wird. Eine starre zeitliche Grenze besteht nicht. §30 I SGB I weist einen doppelten Regelungsgehalt auf: die Norm bestimmt als einseitige Kollisionsnorm, in welchen Fällen der persönliche Anwendungsbereich des deutschen Sozialrechts eröffnet ist und klärt zugleich die örtliche Zuständigkeit der diesem unterliegenden Sozialleistungsträger. ${ }^{6}$ Darüber hinaus soll durch das Abstellen auf die tatsächlichen Verhältnisse eine lediglich formale Wohnsitzbegründung zum Zwecke der Inanspruchnahme von Sozialleistungen vermieden werden. ${ }^{7}$

2 B. v. Maydell, Internationales Sozialversicherungsrecht, in: Festschrift 25 Jahre BSG, Band 2, 1979, S. 943, 947; P. Becker in Hauck/Noftz, SGB I, §30, Rn. 1; R. Schlegel in jurisPKSGB I, §30, Rn. 18; C.-J. Schulte, ZSR 1976, S. 334, 337.

3 B. v. Maydell, VSSR 1973, S. 347, 359; C.-J. Schulte, ZSR 1976, S. 334, 339 ff.; Selb in Tomandl, Auslandsberührungen in der Sozialversicherung, S. 18.

4 BT-Drs. 7/868, 27.

5 Selb in Tomandl, Auslandsberührungen in der Sozialversicherung, S. 23.

6 BSGE 67, 243; Selb in Tomandl, Auslandsberührungen in der Sozialversicherung, S. 23.

7 BT-Drs. 7/3786, 5. 
a) Materielle Rechtmäßigkeit des Aufenthalts als Bedingung der Leistungsberechtigung?

Ob der gewöhnliche Aufenthalt nur im Falle seiner materiellen Rechtmäßigkeit begründet ist, ist fraglich. Der Wortlaut des $\S 30$ III SGB I legt ein Abstellen auf die tatsächlichen Umstände nahe, um einen Sozialleistungsmissbrauch durch eine bloß formale Wohnsitzbegründung zu verhindern.

Nach der vormals von der Rechtsprechung vertretenen Einfärbungslehre ergibt sich die konkrete normative Bedeutung des Aufenthaltsbegriffs erst aus dem Gesetz, das ihn verwendet. Der Aufenthaltsbegriff des $\S 30$ I SGB I werde also durch den jeweiligen Gesetzeszweck sozialleistungsspezifisch „eingefärbt"“. ${ }^{8}$ Das BSG hat diese Rechtsprechung inzwischen zugunsten einer für alle Sozialleistungen einheitlichen Auslegung aufgegeben. ${ }^{9}$ Für die Eröffnung des Anwendungsbereichs des Sozialrechts komme es jedoch nicht allein auf die physische Präsenz auf dem Bundesgebiet an. Der Aufenthalt sei nur dann gewöhnlich, wenn er ausländerrechtlich beständig sei, dem Ausländer also gleich einem Inländer ein zukunftsoffenes, zeitlich unbestimmtes Verweilen in der Bundesrepublik erlaube. Denn ein aufenthaltsrechtlich unzulässiger Aufenthalt dürfe nicht über das Sozialrecht legitimiert und zum Anknüpfungspunkt für Rechtsansprüche gemacht werden. ${ }^{10}$ Der gewöhnliche Aufenthalt fehlt bei den Inhabern einer Aufenthaltsberechtigung nach dem AsylVfG oder einer Duldung. Bei irregulären Migranten, die sich ohne jedweden Aufenthaltstitel in der Bundesrepublik aufhalten, ist - ungeachtet ihrer faktischen Aufenthaltsdauer - die rechtliche Unbeständigkeit angesichts des Erlaubnisvorbehalts und der Ausreisepflicht nach $\S 50$ I AufenthG evident.

\section{b) Sozialhilfebedürftigkeit als Einreisehindernis}

Um einen Aufenthaltstitel erlangen zu können, muss ein Ausländer die in $\S 5$ I, II AufenthG enumerierten Voraussetzungen erfüllen. Unter anderem muss seine Identität geklärt sein, er muss über einen Pass verfügen und mit dem erforderlichen Visum in die Bundesrepublik eingereist sein. Ausweisungsgründe nach $\S \S 53 \mathrm{ff}$. AufenthG dürfen ebenso wenig vorliegen wie sonstige Umstände, die eine Beeinträchtigung oder Gefährdung der Interessen der Bundesrepublik begründen. $\mathrm{Zu}$ den geschützten Interessen zählen alle wirtschaftlichen, gesellschaftlichen und arbeitsmarktpolitischen Interessen. ${ }^{11}$ Die Erteilung eines Aufenthaltstitels setzt daher regelmäßig voraus, dass der Lebensunterhalt des Ausländers gesichert ist, $\S 5$ I Nr. 1 AufenthG. Nur bei den humanitären Aufenthaltstiteln wird von diesem Erfordernis abgesehen, § 5 III AufenthG.

Der Lebensunterhalt gilt als gesichert, wenn der Ausländer hierzu keine öffentlichen Mittel in Anspruch nehmen muss. Dies ist der Fall, wenn er selbst über die notwendigen

8 Zur Familienkrankenhilfe BSGE 57, 93, 95 f.; zum freiwilligen Beitritt zur Krankenversicherung BSGE 60, 262, 264; zum Bundeserziehungsgeld BSGE 62, 67, 71 sowie BSGE 67, 243, 249; zum Kindergeld BSG, SozR 5870 § 1 Nr. 12; zur rentenrechtlichen Relevanz von Kindererziehungszeiten BSG, SozR 3-1200 §30 Nr. 11 sowie BSG, SozR 3-1200 §30 Nr. 21; zur Sozialhilfe für Deutsche im Ausland BVerwGE 99, 158, 162.

9 BSGE 63, 47.

10 Vgl. nur BSGE 65, 261; 80, 209.

11 G. Renner, Ausländerrecht, § 4 AufenthG, Rn. 27; vgl. auch BVerwGE 61, 105, $107 \mathrm{f}$. 
finanziellen Ressourcen verfügt oder diese aus privaten Mitteln Dritter erhält, § 2 III AufenthG. Alternativ kann ein Dritter eine Verpflichtungserklärung nach $\S 68$ AufenthG abgeben. Maßgeblich sind die individuellen Bedürfnisse des einzelnen Ausländers für Ernährung, Wohnraum, Kleidung und Hygiene, nicht aber besondere, außergewöhnliche und unvorhersehbare Belastungen. ${ }^{12}$ Zwingend erforderlich ist auch ein ausreichender Krankenversicherungsschutz, der zumindest der Art nach den in der deutschen Sozialversicherung geltenden Bedingungen entspricht. ${ }^{13}$ Der Höhe nach müssen die Mittel in etwa den Umfang der Regelsätze nach dem SGB II bzw. SGB XII erreichen. ${ }^{14}$ Die Ausländerbehörden haben insoweit eine Prognose zu treffen, denn die Fähigkeit zum Selbstunterhalt darf nicht nur vorübergehender Natur sein. Unschädlich ist der Bezug von Sozialleistungen, denen eigene Beitragsleistungen zugrunde liegen. Wesentlicher Anwendungsbereich des $\S 5$ I Nr. 1 AufenthG ist daher der Sozialhilfebezug. Im Grunde wird der für die Möglichkeit des Zugangs zu Sozialleistungen erforderliche gewöhnliche Aufenthalt daher nur den Personen eröffnet, die über so hinreichende Mittel verfügen, dass sie diese auf absehbare Zeit nicht in Anspruch nehmen!

\section{Erwerbsfähigkeit von Ausländern}

Über den gewöhnlichen Aufenthalt hinaus setzt $§ 7$ I 1 SGB II voraus, dass der Berechtigte erwerbsfähig und hilfebedürftig ist. Ausländer gelten nach $\S 8$ II SGB II als erwerbsfähig, wenn ihnen die Aufnahme einer Beschäftigung erlaubt ist oder erlaubt werden kann.

\section{Zugang zum Arbeitsmarkt}

Dies folgt daraus, dass Ausländern nach $\S 4$ III AufenthG ohne ausdrückliche Erlaubnis die Ausübung einer Erwerbstätigkeit in der Bundesrepublik untersagt ist. Im System des SGB II ist diese Voraussetzung schlüssig, werden doch neben den existenzsichernden Regelleistungen Maßnahmen zur Eingliederung in den Arbeitsmarkt geleistet, § 1 II SGB II. An die Mitwirkung an solchen aktivierenden Maßnahmen sind zudem Sanktionen, beispielsweise die Absenkung der Regelleistung bei Ablehnung einer zumutbaren Arbeit nach § 31 I 1 Nr. 1 c SGB II, geknüpft. Dies liefe ins Leere, wenn dem Berechtigten der Zugang zum inländischen Arbeitsmarkt verschlossen ist.

$\mathrm{Ob}$ der Zugang zum deutschen Arbeitsmarkt eröffnet ist, ergibt sich aus dem AufenthG, auf das § 7 I 3 SGB II verweist. Nach § 9 I 2 AufenthG ist den Inhabern einer Niederlassungserlaubnis kraft Gesetzes uneingeschränkt die Aufnahme einer Erwerbs-

12 EuGH NVwZ 2010, S. 697 (Rs. Chakroun) weist ausdrücklich darauf hin, dass es nur auf den Regelbedarf ankommen kann.

13 So bereits BVerwGE 94, 35, 44 zum AuslG 1990; vgl. auch R. Marx, ZAR 2010, S. 222, 226; G. Renner, Ausländerrecht, § 2 AufenthG, Rn. 16.

14 K. Hailbronner, Asyl- und Ausländerrecht, Rn. 96 m.w.N.; B. Huber/R. Göbel-Zimmermann, Ausländer- und Asylrecht, Rn. 15; VGH Kassel ZAR 2006, S. 145, 146. 
tätigkeit gestattet. ${ }^{15}$ Ausländern, die eine Aufenthaltserlaubnis aus anderen Gründen anstreben, kann gemäß $§ 18$ AufenthG die Erlaubnis zur Erwerbstätigkeit erteilt werden, sofern die Bundesagentur für Arbeit nach Maßgabe des $\$ 39$ AufenthG zustimmt. Die Zustimmung steht jedoch unter dem Vorbehalt, dass für den konkreten Arbeitsplatz keine vorrangig zu berücksichtigenden deutschen oder privilegierten ausländischen Arbeitnehmer - vorwiegend EU-Bürger - zur Verfügung stehen. Asylbewerber und Geduldete haben nach einjährigem gestatteten Aufenthalt in der Bundesrepublik die Möglichkeit, eine Arbeitserlaubnis zu erlangen, § 61 II AsylVfG, § 10 BeschVerfV. Deren Erteilung steht ebenfalls unter dem Vorbehalt der Zustimmung der Bundesagentur für Arbeit einschließlich der in $\S 39$ AufenthG vorgesehenen Vorrangprüfung.

\section{Abstrakte oder konkrete Betrachtung?}

Ob die Möglichkeit der Gestattung der Erwerbstätigkeit abstrakt-generell oder konkret zu beurteilen ist, war lange umstritten. Der Wortlaut des $\S 8$ II SGB II deutete darauf hin, dass lediglich auf die im AufenthG und anderen migrationsrechtlichen Vorschriften eröffnete Möglichkeit zur Erteilung einer Beschäftigungserlaubnis abzustellen ist. ${ }^{16}$ Vereinzelt wurde jedoch eine konkrete Betrachtung gefordert. Es müsse eine realistische Chance auf Erteilung einer Beschäftigungserlaubnis bestehen, sodass es auf das tatsächliche Vorhandensein einer Arbeitsgelegenheit für den Ausländer ankomme, für die auch keine vorrangig zu berücksichtigenden Arbeitnehmer zur Verfügung stehen. ${ }^{17}$ Die konkrete Betrachtungsweise führte jedoch faktisch zum Ausschluss von sämtlichen nichtprivilegierten Ausländern aus dem Leistungssystem des SGB II. Besteht eine konkrete Arbeitsgelegenheit, so mag nach der konkreten Betrachtungsweise zwar die Erwerbsfähigkeit des Antragstellers vorliegen. Hilfebedürftig i.S.v. § 9 SGB II wird er dann im Allgemeinen jedoch nicht mehr sein, da ihm ein konkretes Erwerbseinkommen zusteht, das er zum eigenen Lebensunterhalt verwerten kann. Er steht also dem deutschen Arbeitsmarkt bereits zu nah! Die Differenzierung in § 8 II SGB II kann sich daher nur auf das „Erlaubtsein“ der Erwerbstätigkeit kraft Gesetzes und das abstrakte „Erlaubtwerdenkönnen“ der Erwerbstätigkeit im Inland nach $\S § 18,39$ AufenthG beziehen. ${ }^{18}$ Mit Wirkung vom 1.4.2011 ist dies durch die Aufnahme von $\S 8$ II 2 SGB II klargestellt worden. Aufgrund von § 8 II SGB II sind daher lediglich Inhaber eines Visums nach $\S 6$ AufenthG, also Touristen oder Durchreisende, sowie Asylbewerber und

15 Gleiches gilt für die Inhaber einer Daueraufenthaltserlaubnis-EG (§ 9 a I 2 AufenthG), einer Aufenthaltserlaubnis zum Zwecke der Ausbildung ( 16 III AufenthG), einer Aufenthaltserlaubnis für Asylberechtigte oder anerkannte Flüchtlinge ( $\$ 25$ I, II AufenthG) oder Familiennachzügler ( $\S 28 \mathrm{~V}, 29 \mathrm{~V}$ AufenthG).

16 So auch die Anwendungshinweise der Bundesagentur für Arbeit zu $§ 8$ SGB II, Rn. 8.15 (www.arbeitsagentur.de).

17 LSG Berlin-Brandenburg, Beschluss vom 13.12.2005 (L 25 B 1281/05 AS ER)-juris; LSG Rheinland-Pfalz FEVS 58, 403; LSG Niedersachsen NZS 2007, S. 431, 432; LSG BadenWürttemberg NZS 2009, S. 512, 514; LSG Berlin-Brandenburg, Beschluss vom 5.5.2009 (L 29 AS 396/09 B), so offenbar auch H. M. Heinig, ZESAR 2008, S. 465 (Fn. 34); R. Seegmüller in Estelmann, SGB II, § 8, Rn. 45; J. Blüggel in Eicher/Spellbrink, SGB II, § 8, Rn. 66 ff.

18 Im Ergebnis so auch K. Sieveking, ZAR 2004, S. 283, 286; M. Gerenkamp in Mergler/Zink, $\S 7$ SGB II, Rn. 11. 
Geduldete im ersten Jahr ihres Aufenthalts von vornherein vom Bezug der Grundsicherung ausgeschlossen.

\section{Sonderregelungen im Leistungsbezug für Ausländer}

Der Gesetzgeber hat in $§ 7$ I 2 SGB II weitere Sonderregelungen für Ausländer etabliert, mit denen diese trotz eines gewöhnlichen Aufenthalts in der Bundesrepublik von den Leistungen der Grundsicherung für Arbeitsuchende ausgeschlossen werden.

\section{Leistungsausschluss während der ersten drei Monate des Aufenthalts}

Gemäß $§ 7$ I 2 Nr. 1 SGB II haben sämtliche Ausländer und deren Familienangehörige während der ersten drei Monate ihres Aufenthalts keinen Anspruch auf Leistungen nach dem SGB II. Dies ist selbst dann der Fall, wenn sie einen auf Dauer angelegten Aufenthalt in der Bundesrepublik begründen. Die Klausel statuiert damit eine Wartezeit. Erst nach deren Ablauf können gewöhnlich sich in Deutschland aufhaltende Ausländer Leistungen der Grundsicherung in Anspruch nehmen.

Etwas anderes gilt nur, wenn sich Ausländer als Arbeitnehmer oder Selbständige im Inland aufhalten, selbst wenn sie - so die Verweisung auf $\S 2$ III FreizügG/EU - vorübergehend erwerbsgemindert oder arbeitslos sind oder sich in der Berufsausbildung befinden. Inhaber einer Aufenthaltserlaubnis aus politischen, völkerrechtlichen oder humanitären Gründen nach $\S \S 22-26$ AufenthG sind vom Leistungsausschluss ebenfalls nicht betroffen, § 7 II 3 SGB II. Der Zweck der Ausschlussklausel, keinen Anreiz für Migration aus wirtschaftlichen Gründen zu bieten, steht bei dieser Personengruppe offenkundig nicht im Vordergrund ihrer Zuwanderungsentscheidung. ${ }^{19}$

\section{Leistungsausschluss wegen Aufenthalts zur Beschäftigungssuche}

Nach Ablauf der dreimonatigen Wartefrist kommt es für die Leistungsberechtigung nach dem SGB II auf den Zweck des Aufenthalts an. Gemäß § 7 I 2 Nr. 2 SGB II sind Personen, deren Aufenthalt allein der Beschäftigungssuche dient, generell von den Leistungen nach SGB II ausgeschlossen.

Der Leistungsausschluss betrifft Unionsbürger, die von ihrer Freizügigkeit aus Art. 45 AEUV Gebrauch machen und auf dem Gebiet der Bundesrepublik eine Beschäftigung suchen, aber auch Angehörige der EWR-Staaten und der Schweiz, die der EU mit einem Freizügigkeitsabkommen verbunden sind. Drittstaatsangehörigen ist der Aufenthalt ausschließlich zur Beschäftigungssuche allein nach § 16 IV AufenthG gestattet, d.h. nach erfolgreichem Abschluss eines Studiums im Inland, allerdings begrenzt auf die Dauer eines Jahres.

19 F. Schreiber, info also 2008, S. 3, 3; A. Brühl/D. Schoch in Münder, LPK-SGB II, § 7, Rn. 40. 


\section{Leistungsausschluss wegen Leistungsbezugs nach AsylbLG}

Ein weiterer genereller Leistungsausschluss betrifft die Bezieher von Leistungen nach dem AsylbLG, § 7 I 2 Nr. 3 SGB II. Das AsylbLG begründet ein Sondersystem der Sozialhilfe für Personen, deren Aufenthalt in der Bundesrepublik lediglich vorübergehender Natur ist. Die ihnen zu gewährenden Leistungen zur Sicherung des Lebensunterhalts sind in diesem Gesetz abschließend geregelt, da das Ziel der langfristigen Eingliederung in den Arbeitsmarkt bei dieser Personengruppe aufgrund ihres unsicheren Aufenthaltsstatus nicht erreichbar sei. ${ }^{20}$

\section{Zulässigkeit der Differenzierung nach der Staatsangehörigkeit}

Der gestufte Zugang von Ausländern zur Grundsicherung für Arbeitsuchende scheint auf den ersten Blick konsequent und deckungsgleich mit aufenthaltsrechtlichen Wertungen. Personen, deren Aufenthalt weniger als drei Monate andauert, begründen nicht zwingend und in jedem Fall ihren Lebensmittelpunkt in der Bundesrepublik. ${ }^{21}$

Das deutsche Verfassungsrecht lässt im Rahmen des allgemeinen Gleichheitssatzes aus Art. 3 I GG Spielraum für Ungleichbehandlungen zwischen Deutschen und Ausländern, wenn diese auf einem objektiv tragfähigen Differenzierungsgrund beruhen. ${ }^{22}$ Ein völliger Ausschluss vom Zugang zu existenzsichernden Sozialleistungen verstößt aber gegen das aus Art. 1 I, 20 I GG hergeleitete Grundrecht auf Sicherung einer menschenwürdigen Existenz, ${ }^{23}$ wenn der Betreffende keine Möglichkeit hat, einen Anspruch auf Gewährung hinreichender Mittel zur Sicherung seines Lebensunterhalts geltend zu machen. Bei Ausländern besteht insofern - anders als bei den eigenen Staatsangehörigen - zumindest dem Grunde nach die Möglichkeit, den Bedürftigen der Verantwortung eines anderen Staats, i.e. seines Heimat- bzw. Herkunftsstaats, zuzuweisen. ${ }^{24}$ Dies bedarf einer Überprüfung der Bindungen und Bezugspunkte des Betroffenen zum Aufenthalts- sowie zum Herkunftsstaat, um abschließend über einen Statutenwechsel befinden zu können. Ob diese Überlegung eine hinreichende Basis für staatsbürgerschaftsbezogene Differenzierungen im Grundsicherungsrecht zu bilden vermag, sei im Folgenden anhand der europa- und völkerrechtlichen Gleichbehandlungsgebote untersucht.

\section{Primärrechtliche Differenzierungsverbote}

Das Recht auf Gleichbehandlung ist für Arbeitnehmer in Art. 45 II AEUV, für alle anderen Unionsbürger in Art. 18 AEUV verankert.

20 M. Gerenkamp in Mergler/Zink, § 7 SGB II, Rn. 19. Ausführlich zum AsylbLG C. Janda/F. Wilksch, SGb 2010, S. 565.

21 So auch K. Strick, NJW 2005, S. 2182, 1285.

22 BVerfGE 55, 72; 88, 87; 112, 368; 116, 229.

23 BVerfGE 125, 175.

24 E. Eichenhofer, SGb 2011, S. 463, 466; so wohl auch M. Husmann, NZS 2009, S. 652, 654; LSG Niedersachsen-Bremen, Beschl. v. 26.2.2010 (L 15 AS 30/10 B ER)-juris. 
a) Gleichbehandlungsansprüche der Arbeitnehmer, Art. 45 AEUV

Die Arbeitnehmerfreizügigkeit ist auch Personen eröffnet, die in einem anderen Mitgliedstaat Arbeit suchen. Sie haben das Recht, sich vom Wohnstaat aus auf Stellen zu bewerben, können zu diesem Zweck aus Art. 45 I AEUV aber auch ein Recht auf Einreise in einen anderen Mitgliedstaat ableiten.

Art. 45 II AEUV garantiert die Abschaffung jeder auf der Staatsangehörigkeit beruhenden unterschiedlichen Behandlung der Arbeitnehmer der Mitgliedstaaten in Bezug auf Beschäftigung, Entlohnung und sonstige Arbeitsbedingungen. Nach der Entscheidung des Europäischen Gerichtshofs (EuGH) in der Rechtssache Collins erfordert dies aber eine „tatsächliche Verbindung“ des Arbeitsuchenden zu dem Arbeitsmarkt des Aufenthaltsstaats. In diesen Fällen ist absolute Gleichbehandlung mit Inländern zu gewährleisten, und zwar auch im Hinblick auf finanzielle Leistungen, die den Zugang zum Arbeitsmarkt erleichtern sollen. ${ }^{25}$ Der Europäische Gerichtshof hat insoweit seine frühere Rechtsprechung ${ }^{26}$ aufgegeben, nach der sich die Gleichbehandlungsansprüche nur auf Arbeitnehmer, nicht aber auf Arbeitsuchende erstrecken. Dieser in den 1980er Jahren begründeten Rechtsprechung lag der damalige Rechtszustand zugrunde, der Freizügigkeitsrechte an die wirtschaftliche Aktivität koppelte und der durch den MaastrichtVertrag überwunden worden ist.

Der Gleichbehandlungsanspruch aus Art. 45 II AEUV gilt absolut. Differenzierungen nach der Staatsangehörigkeit eines Arbeitnehmers sind daher keiner Rechtfertigung zugänglich. Das vom Europäischen Gerichtshof aufgestellte Kriterium der tatsächlichen Verbindung stellt dementsprechend auch kein rechtfertigendes Element für Ungleichbehandlungen dar, sondern statuiert ein Anknüpfungsmoment für die Zuständigkeit des Aufenthaltsstaats und die Eröffnung des Gleichbehandlungsgebots aus Art. 45 II AEUV. Insofern ist es deckungsgleich mit dem im deutschen Recht aufgestellten Erfordernis des gewöhnlichen Aufenthalts: nur Arbeitsuchende mit gewöhnlichem Aufenthalt in der Bundesrepublik können sich auf die Verantwortlichkeit des deutschen Sozialleistungssystems berufen und sind dort gleich zu behandeln. ${ }^{27}$ Dies deckt sich im Ergebnis auch mit dem Arbeitnehmerbegriff des Europäischen Gerichtshofs. ${ }^{28}$ Das Gericht differenziert zwischen Unionsbürgern, die sich als Arbeitsuchende erstmalig in einen anderen Mitgliedstaat begeben und solchen, die bereits in einem anderen Mitgliedstaat beschäftigt waren und dort arbeitslos werden. Nur letzteren räumt es einen umfassenden Gleichbehandlungsanspruch ein. Alle anderen Unionsbürger erfüllen dieses ungeschriebene Tatbestandsmerkmal des Art. 45 II AEUV nicht. Ihr Aufenthalt fällt in den Anwendungsbereich der Unionsbürgerfreizügigkeit nach Art. 18 AEUV.

25 EuGH, Slg. 2004, I-2703, Rn. 63 (Collins), bestätigt in Slg. 2005, I-8275, Rn. 22 (Ioannidis) sowie Slg. 2009, I-4585, Rn. 37 (Vatsouras und Koupatantze).

26 EuGH, Slg. 1987, 2811, Rn. 26 (Lebon).

27 Im Ergebnis auch F. Schreiber, ZESAR 2006, S. 424, 429; M. Husmann, NZS 2009, S. 652, 656; E. Eichenhofer, SGb 2011, S. 463, 465.

28 EuGH, Slg. 1982, 1035, Rn. 18 (Levin); Slg. 1986, 1741, Rn. 16 (Kempf); Slg. 1988, 3161, Rn. 33 (Lair); Slg. 2004, I-2703, Rn. 32 (Collins). 
b) Gleichbehandlungsansprüche freizügigkeitsberechtigter Unionsbürger, Art. 18 AEUV

Mit der Unionsbürgerfreizügigkeit nach Art. 21 AEUV ist auch den wirtschaftlich Inaktiven das Recht zum Aufenthalt und zur Beschäftigungssuche in anderen Mitgliedstaaten eingeräumt. ${ }^{29}$ Die Etablierung von Wartezeiten und darüber hinaus die Versagung existenzsichernder Leistungen während der Arbeitssuche könnte die Ausübung dieses Rechts unzulässig beeinträchtigen und zugleich dem Diskriminierungsverbot aus Art. 18 AEUV zuwiderlaufen.

\section{(1) Rechtfertigung der Ungleichbehandlung durch fiskalische Interessen?}

Wohnsitz- oder Aufenthaltserfordernisse und Wartezeiten sollen einer einseitigen Belastung der Sozialleistungssysteme einzelner Mitgliedstaaten entgegenwirken. Eine solche könne durch einen uneingeschränkten Zugang zu den existenzsichernden Leistungen ausgelöst werden, bestehe in diesem Fall doch ein Anreiz für Sozialtourismus. Die Ausschlussklauseln sind also von dem Anliegen getragen, nur diejenigen an steuerfinanzierten Leistungen teilhaben zu lassen, die bereits selbst einen Beitrag zum gesamtgesellschaftlichen Wohlstand geleistet haben. Halte sich ein Ausländer erst seit Kurzem im Inland auf, sei er noch nicht in die Gesellschaft integriert und habe dieser gegenüber noch keinerlei Leistungen erbracht. Daher sei er noch nicht als Bürger dieser Solidargemeinschaft anzusehen. ${ }^{30}$

Führt man diesen Gedanken konsequent fort, bedeutet dies, dass nur ehemaligen Erwerbstätigen - denn nur diese tragen durch Erfüllung ihrer steuerlichen Verpflichtungen zur Finanzierung der Sozialhilfe bei - die hinreichende Nähe zum Aufenthaltsstaat zukommt, aus der sich deren Teilhabeansprüche ableiten lassen sollen. Wer nicht am Wirtschaftsleben teilnehme, müsse selbst für sein Auskommen sorgen und könne keine Inklusion in die sozialen Sicherungssysteme seines Aufenthaltsstaats fordern. ${ }^{31}$

\section{(2) Wirtschaftliche Leistungsfähigkeit als Aufenthaltsvoraussetzung}

Diese Ansicht scheint im Sekundärrecht zunächst eine Stütze zu finden, wird die Reichweite der Unionsbürgerfreizügigkeit durch die Unionsbürgerrichtlinie doch insoweit beschränkt, als Nichterwerbstätige wirtschaftlich leistungsfähig sein müssen, um sich auf ihr Freizügigkeitsrecht berufen zu können. Das kurzfristige Aufenthaltsrecht besteht nach Art. 14 I RL 2004/38/EG nur, solange Sozialhilfeleistungen ,nicht unangemessen“ in Anspruch genommen werden. Einen Aufenthalt von mehr als drei Monaten dürfen wirtschaftlich Inaktive nur begründen, wenn sie hinreichende Existenzmittel nachweisen, die eine Inanspruchnahme öffentlicher Leistungen unwahrscheinlich machen, Art. 7 I lit. b) RL 2004/38/EG. Erst das Daueraufenthaltsrecht nach fünfjährigem rechtmäßigem Aufenthalt entsteht gemäß Art. 16 I 2 RL 2004/38/EG unabhängig von der wirtschaftlichen Leistungsfähigkeit.

29 EuGH, Slg. 1996, I-2617 (O’Flynn).

30 M. Windisch-Graetz in Eilmansberger/Herzig, Soziales Europa, S. 100.

31 So C. Tomuschat, CML Rev 37 (2000), S. 449, 455; I. Niemann, EuR 2004, S. 946, 947. 
Indes folgt aus der Systematik der Unionsbürgerrichtlinie, dass das Fehlen der notwendigen Mittel zum Lebensunterhalt nicht bereits Voraussetzung für die Begründung des Aufenthaltsrechts, sondern erst für dessen Fortbestand von Relevanz ist. ${ }^{32}$ Für die Begründung des Aufenthalts in einem anderen Mitgliedstaat bedarf es keines förmlichen Verfahrens, in dem ein Titel erteilt wird. Art. 14 II RL 2004/38/EG statuiert, dass die Aufenthaltsrechte der Unionsbürger und ihrer Familienangehörigen bestehen, solange diese die in Art. 7 RL 2004/38/EG statuierten Voraussetzungen erfüllen. Auch $\S 5$ III FreizügG/EU ermöglicht eine Überprüfung der Aufenthaltsvoraussetzungen erst nach Ablauf von drei Monaten. Nach $\S 5$ V FreizügG/EU kann bei Entfallen der Aufenthaltsvoraussetzungen ex post der Verlust des Aufenthaltsrechts festgestellt werden.

Ein Antrag auf Leistungen zur Sicherung des Lebensunterhalts deutet zwar darauf hin, dass die Voraussetzungen der Freizügigkeit entfallen sind. Dies hat aber nicht per se die Rechtswidrigkeit und damit die zwangsweise Beendigung des Aufenthalts zur Folge. Der Europäische Gerichtshof hat wiederholt darauf hingewiesen, dass es den Mitgliedstaaten zwar aufgrund der sekundärrechtlichen Regelungen unbenommen ist, einem bedürftigen Unionsbürger, der keiner Erwerbstätigkeit nachgeht, den Aufenthalt zu verweigern. Sie dürfen diese Befugnis jedoch nur unter Beachtung des Verhältnismäßigkeitsprinzips ausüben. Die Ausweisung dürfe demnach keine automatische Folge der Bedürftigkeit sein. ${ }^{33}$ Art. 14 IV RL 2004/38/EG verbietet generell die Ausweisung von Arbeitnehmern und Selbständigen (lit. a) und von Arbeitsuchenden und deren Familienangehörigen, solange diese ernsthaft und mit Aussicht auf Erfolg nach einer Beschäftigung suchen (lit. b). Die Ausweisung kommt daher nur in Ausnahmefällen in Betracht. In jedem Fall kann die Rechtmäßigkeit des Aufenthalts erst nach Ergehen einer bestandskräftigen Ausweisungsverfügung verneint werden. ${ }^{34}$ Es gilt mithin eine Vermutung der Freizügigkeit, die nur durch behördliche Feststellung entkräftet werden kann..$^{35}$

\section{(3) Statutenwechsel als Voraussetzung der Gleichbehandlung}

Eine die Freizügigkeit hindernde Ausweisungsverfügung gegen Angehörige anderer Mitgliedstaaten stellt als solche keinen Verstoß gegen das Diskriminierungsverbot aus Art. 18 AEUV dar. Zwar können denknotwendig nur fremde Staatsangehörige davon betroffen sein. Die Ausweisung aus Gründen der Bedürftigkeit ist jedoch gerechtfertigt, wenn die Zuständigkeit für die Sicherung der Existenz einem anderen Mitgliedstaat, i.e. dem Herkunftsstaat, zufällt.

32 M. Husmann, NZS 2009, S. 652, 653; K. Dienelt in Renner, Ausländerrecht, § 4 FreizügG/ EU, Rn. 37; C. Schönberger, Unionsbürger, S. 375.

33 EuGH, Slg. 2001, I-6193, Rn. 42 f. (Grzelczyk); Slg. 2002, I-7091, Rn. 87 ff. (Baumbast); Slg. 2004, I-7573, Rn. 45 (Trojani).

34 F. Schreiber, ZESAR 2006, S. 424, 429; W. Frenz/A. Kühl, ZESAR 2007, S. 313, 320; P.-C. Kunkel/M. Frey, ZFSH/SGB 2008, S. 387, 393; K. Hailbronner, ZFSH/SGB 2009, S. 195, 197 f.; F. Schreiber, info also 2009, S. 195, 199; LSG Baden-Württemberg NZS 2009, S. 512, 515; a.A. unter Berufung auf den Willen des Gesetzgebers SG Reutlingen, Urt. v. 29.4.2008 (S 2 AS 2952/07)-juris.

35 BT-Drs. 15/420, 106. 
Im Umkehrschluss begründet der einem Unionsbürger gewährte Aufenthalt einen umfassenden Anspruch auf Teilhabe an den Leistungen des Sozialsystems, welches von den die Rechtmäßigkeit des Aufenthalts bedingenden Voraussetzungen entkoppelt ist. ${ }^{36}$ Diesen Befund spiegelt die Rechtsprechung des Europäischen Gerichtshofs wieder: die Unionsbürgerschaft gewähre dem Einzelnen einen ,grundlegenden Status“, der ihm unabhängig von seiner Staatsangehörigkeit die gleichen Rechte verleihe, die auch die Angehörigen des Aufenthaltsstaats genießen. ${ }^{37}$ Das Interesse der Mitgliedstaaten, die finanzielle Tragfähigkeit ihrer sozialen Sicherungssysteme zu schützen, sei nur dann geschützt, wenn die finanziellen Belastungen erheblich seien. Zudem müsse auch im Einzelfall die Verhältnismäßigkeit gewahrt sein: habe ein Unionsbürger bereits seit einigen Jahren seinen Lebensmittelpunkt in einem anderen Mitgliedstaat und bedürfe er für einen lediglich vorübergehenden Zeitraum der Leistungen der Sozialhilfe, sei eine solche Belastung ,über Gebühr“ noch nicht gegeben. Je länger der Aufenthalt besteht, umso weiter ist die Inklusion in die Gesellschaft des Aufenthaltsstaats fortgeschritten. Die Mitgliedstaaten können also nicht einerseits Freizügigkeit gewähren, andererseits aber Solidarität gegenüber den auf ihrem Hoheitsgebiet befindlichen Personen verweigern. ${ }^{38}$

\section{c) Wirksamkeit der dreimonatigen Wartezeit}

Daraus ist jedoch nicht zwingend auf die Unwirksamkeit der Ausschlussklausel in $\S 7$ I 2 Nr. 1 SGB II zu schließen. Wartezeiten sind vom Europäischen Gerichtshof grundsätzlich gebilligt worden, dienen sie doch dem Nachweis einer tatsächlichen Verbindung des Antragstellers zu dem die Leistung gewährenden Staat und sind daher geeignet, Sozialtourismus zu vermeiden. Es ist jedoch fraglich, ob die in $\S 7$ I 2 Nr. 1 SGB II verankerte Wartezeit erforderlich ist, wenn sie auch Personen mit gewöhnlichem Aufenthalt in der Bundesrepublik zurücklegen müssen. Der gewöhnliche Aufenthalt, der durch eine zukunftsoffene Verlagerung des Lebensmittelpunkts gekennzeichnet ist, bei der eine Rückkehr ins Herkunftsland nicht absehbar ist, ist für sich genommen bereits geeignet, eine tatsächliche Bindung zum Aufenthaltsstaat zu begründen. Denn auch wirtschaftlich Inaktive können unabhängig von sonstigen anerkannten Aufenthaltszwecken einen gewöhnlichen Aufenthalt in der Bundesrepublik begründen. Ist dessen Rechtmäßigkeit nicht in einem förmlichen Verfahren abgelehnt worden, ist Gleichbehandlung zu gewährleisten.

Vor diesem Hintergrund ist die starre dreimonatige Wartefrist als unverhältnismäßig einzustufen, ermöglicht sie es doch nicht, auf Besonderheiten des Einzelfalls einzugehen. ${ }^{39}$ Unionsbürger, die ihren gewöhnlichen Aufenthalt zukunftsoffen und zeitlich unbegrenzt in der Bundesrepublik begründet haben, müssen um ihrer Gleichbehandlungsansprüche aus Art. 21, 18 AEUV willen daher ab dem ersten Tag ihres Aufenthalts unter den gleichen Bedingungen wie Deutsche Zugang zur Grundsicherung bekommen. Diese

36 H. M. Heinig, ZESAR 2008, S. 465, 468.

37 EuGH, Slg. 1998, I-2691 (Martínez Sala); kritisch U. Becker, EuR 1999, S. 522, 531 f.; EuGH, Slg. 2001, I-6193 (Grzelczyk), dazu kritisch K. Hailbronner, NJW 2004, S. 2185, $2186 \mathrm{f}$.

38 EuGH, Slg. 2001, I-6193 (Grzelczyk); Slg. 2005, 2119 (Bidar).

39 So auch M. Husmann, NZS 2009, S. 652, 656. 
Begründung des Lebensmittelpunkts als Anspruchsvoraussetzung fordert bereits $\S 7$ I 1 Nr. 4 SGB II!

d) Wirksamkeit des vollständigen Leistungsausschlusses für Arbeitsuchende

Auch der in $§ 7$ I 2 Nr. 2 SGB II verankerte Leistungsausschluss ist vor diesem Hintergrund als unverhältnismäßig zu bewerten. Zwar hat es der Europäische Gerichtshof als zulässig erachtet, die Gewährung von Leistungen zur Eingliederung in den Arbeitsmarkt an die Voraussetzung zu knüpfen, dass der Empfänger eine hinreichende Verbindung zum Arbeitsmarkt des Aufenthaltsstaats aufweist. ${ }^{40}$ Diese Verbindung ist jedoch von vornherein bei allen Personen begründet, die ihren gewöhnlichen Aufenthalt in der Bundesrepublik haben. Die vom Europäischen Gerichtshof geforderte Nähe zum Arbeitsmarkt des Aufenthaltsstaats statuiert mithin keinen Rechtfertigungsgrund für Differenzierungen nach der Staatsangehörigkeit, sondern ist konstitutiv für den Statutenwechsel.

Im Ergebnis hat die Rechtsprechung daher nur bestätigt, dass der gewöhnliche Aufenthalt ein geeignetes Anknüpfungskriterium für die Zuständigkeit und Verantwortlichkeit eines wohlfahrtsstaatlichen Systems ist. Damit wird keineswegs dem befürchteten Sozialtourismus arbeitsuchender Unionsbürger Vorschub geleistet. Denn typischerweise wird der Wohnsitz bzw. der Ort des gewöhnlichen Aufenthalts erst dann in einen anderen Staat verlegt, wenn dort ein neues Beschäftigungsverhältnis begründet wird. Die Beschäftigungssuche begründet üblicherweise noch keinen Statutenwechsel, der die Verantwortung für die Gewährung existenzsichernder Leistungen auf einen anderen Staat übergehen lässt. ${ }^{41}$ Solange aber die ,tatsächliche Verbindung“ zum Herkunftsland fortbesteht, besteht kein Anspruch auf Gleichbehandlung mit den Angehörigen des Aufenthaltsstaats.

Der dauerhafte Ausschluss von Arbeitsuchenden in § 7 I 2 Nr. 2 SGB II hält den vom Europäischen Gerichtshof etablierten Kriterien daher nicht stand. Er ist weder geeignet noch erforderlich, um eine tatsächliche Verbindung zum Arbeitsmarkt des Aufenthaltsstaats nachzuweisen, sondern macht es einem arbeitsuchenden Unionsbürger von vornherein unmöglich, diesen Nachweis zu erbringen. ${ }^{42}$

\section{Zulässigkeit der Differenzierung nach Sekundärrecht}

Der Gesetzgeber wollte mit der Einfügung der Leistungsausschlüsse in § 7 SGB II von der Öffnungsklausel des Art. 24 II RL 2004/38/EG Gebrauch machen, die die Gewährung von Leistungen der Sozialhilfe in den ersten drei Monaten des Aufenthalts oder an Arbeitsuchende in das Ermessen der Mitgliedstaaten stellt.

40 EuGH, Slg. 2002, I-6191, Rn. 38 (D’Hoop); Slg. 2004, I-2703, Rn. 70 (Collins); Slg. 2005, I-8275, Rn. 30 (Ioannidis); Slg. 2009, I-4585, Rn. 38 (Vatsouras und Koupatantze).

41 E. Eichenhofer, SGb 2011, S. 463, 465; so auch U. Fasselt, ZFSH/SGB 2004, S. 655, 667; zu den Schwierigkeiten bei der Bestimmung des gewöhnlichen Aufenthalts von Arbeitsuchenden vgl. auch F. Schreiber, info also 2009, S. 195, 198.

42 F. Schreiber, ZESAR 2006, S. 424, 430; M. Husmann, NZS 2009, S. 652, 656. 
a) Leistungen der Grundsicherung als „Sozialhilfe“ i.S.d. Unionsbürgerrichtlinie

Es ist jedoch fraglich, ob diese die Sonderregelungen für Ausländer in der Grundsicherung für Arbeitsuchende trägt. Der Europäische Gerichtshof hat darauf hingewiesen, dass Leistungen, die dem Berechtigten die Eingliederung in den Arbeitsmarkt erleichtern sollen, nicht als Sozialhilfe i.S.v. Art. 24 II RL 2004/38/EG qualifiziert werden können. Die Einstufung einzelner Sozialleistungen richte sich zwar nach nationalem Recht, könne aber nicht anhand formeller Kriterien erfolgen, sondern müsse auf das Ziel und die Voraussetzungen der Leistungsgewährung Bezug nehmen. Dabei deute das Erfordernis der Erwerbsfähigkeit in § 7 I 1 Nr. 2 SGB II darauf hin, dass die Grundsicherung für Arbeitsuchende den Zugang zur Beschäftigung erleichtern solle. Von solchen Leistungen zur Eingliederung in den Arbeitsmarkt können Unionsbürger im Hinblick auf Art. 45 AEUV nicht dauerhaft ausgeschlossen werden. ${ }^{43}$

Nach deutscher Doktrin ist die Grundsicherung für Arbeitsuchende als Leistung der sozialen Hilfen einzustufen: sie wird aus Steuern finanziert und eine wesentliche Funktion ist die Sicherung der Existenz, die über pauschal bemessene Regelsätze sichergestellt wird. Anders als die Leistungen des SGB XII ist die Grundsicherung für Arbeitsuchende jedoch nicht allein vom Fürsorgegedanken geprägt, sondern zielt auch auf die Eingliederung in den Arbeitsmarkt, vgl. § 1 I 2 SGB II. Diese besondere Zielsetzung des SGB II war wegbereitend für die unter Geltung des BSHG nicht erfolgte Einteilung in Leistungen für Erwerbsfähige und Nichterwerbsfähige. Sie kommt an mehreren Stellen im Gesetz zum Ausdruck, wird aber durch das in $\S 2$ SGB II verankerte Prinzip des Förderns und Forderns besonders augenfällig. Die Leistungsbezieher sollen alle Möglichkeiten nutzen, um ihre Hilfebedürftigkeit insbesondere durch Einsatz und Verwertung ihrer Arbeitskraft zu beenden. Zu diesem Zweck sollen die Behörden nach § 3 I 1 SGB II Leistungen zur Eingliederung in den Arbeitsmarkt erbringen. Verstöße gegen die Eingliederungsbemühungen werden nach $\S 31$ SGB II auf der Leistungsebene sanktioniert. Die in der Gesetzessystematik angelegte starke Verknüpfung der Lebensunterhaltssicherung mit den Bemühungen zur Integration in den Arbeitsmarkt spricht dagegen, die Leistungen nach dem SGB II als klassische Sozialhilfe einzustufen. ${ }^{44}$

b) Gleichbehandlungsanspruch aus dem koordinierenden Sozialrecht

Das europäische koordinierende Sozialrecht bestätigt diesen Befund. Dessen Anwendungsbereich ist eröffnet, wenn Leistungen im Hinblick auf eines der in Art. 3 I VO (EG) 883/2004 genannten sozialen Risiken erbracht werden.

43 EuGH, Slg. 2004, I-2703, Rn. 72 f. (Collins) zur britischen Job Seekers' Allowance; EuGH, Slg. 2009, I-4585, Rn. 43 ff. (Vatsouras und Koupatantze) explizit zur Grundsicherung für Arbeitsuchende.

44 M. Fuchs, NZS 2007, S. 1, 4. 


\section{(1) Grundsicherung für Arbeitsuchende im koordinierenden Sozialrecht}

Die Arbeitslosigkeit ist als in die Koordinierung einbezogener Leistungszweig explizit aufgeführt. Dem Risiko der Arbeitslosigkeit sind jedoch nur solche Leistungen zugeordnet, die Einkommensausfälle infolge von Arbeitslosigkeit ausgleichen sollen. Die Grundsicherung für Arbeitsuchende als Hybrid zwischen existenzsichernden und arbeitsmarktbezogenen Leistungen ist darunter nicht fassbar. Auf Existenzsicherung gerichtete Leistungen sind gemäß Art. 3 V VO (EG) 883/2004 explizit von der Koordinierung ausgenommen. Ihr Anliegen, Armut als soziales Risiko zu kompensieren, gilt als traditionelle Aufgabe des Wohn- bzw. Herkunftsstaats, sodass namentlich der Export in andere Mitgliedstaaten nicht angezeigt ist. ${ }^{45}$ Sie stellen daher keine Leistungen der sozialen Sicherheit im Sinne des koordinierenden Sozialrechts dar. Wegen ihrer Anknüpfung an das Merkmal der Erwerbsfähigkeit und ihrer Ausrichtung auf die Beseitigung von Hilfebedürftigkeit durch (Wieder-)Eingliederung in den Arbeitsmarkt, sind die Leistungen der Grundsicherung andererseits auch nicht eindeutig den Fürsorgeleistungen zuzuordnen.

Der Verordnungsgeber hat der Einführung solcher Leistungen mit Mischcharakter Rechnung getragen und in Art. 70 VO (EG) 883/2004 spezifische Regelungen für besondere beitragsunabhängige Geldleistungen etabliert. Darunter sind alle Leistungen zu subsumieren, die an eines der in Art. 3 I VI (EG) 883/2004 genannten sozialen Risiken anknüpfen und ihrem Empfänger ein Mindesteinkommen garantieren, das sich an den im Aufenthaltsstaat geltenden wirtschaftlichen und sozialen Umständen orientiert. Sie sind notwendig aus Steuern zu finanzieren und in ihrer Gewährung unabhängig von früheren Beitragszahlungen (II lit. b) und müssen im Anhang X zur Koordinierungsverordnung enumeriert sein (II lit. c). Die Leistungen zur Sicherung des Lebensunterhalts in der Grundsicherung für Arbeitsuchende erfüllen diese Kriterien. ${ }^{46}$

\section{(2) Sachlicher und personeller Gehalt des Gleichbehandlungsanspruchs}

Zwar unterliegen die besonderen beitragsunabhängigen Geldleistungen nur eingeschränkt den Koordinierungsregeln. Namentlich ist nach Art. 70 III VO (EG) 883/2004 ihr Export ausgeschlossen, denn sie sind an den Wohnstaat und die für diesen geltenden Bestimmungen gebunden. Nicht tangiert davon ist dagegen das Gleichbehandlungsgebot aus Art. 4 VO (EG) 883/2004. ${ }^{47}$ Dabei handelt es sich um eine absolute Garantie der Inländergleichbehandlung, nicht lediglich um ein Diskriminierungsverbot, welches die Möglichkeit der Rechtfertigung von Sonderregelungen eröffnen würde. ${ }^{48}$ Wartefristen wie in $\S 7$ I 2 Nr. 1 SGB II oder der Leistungsausschluss während der Beschäftigungssuche nach $\S 7$ I 2 Nr. 2 SGB II wären daher nur dann zulässig, wenn sie uneingeschränkt auch für Deutsche gelten würden. Die Sonderregelungen des SGB II für Ausländer laufen diesem unbedingten Gleichbehandlungsgebot zuwider.

45 A. Otting in Hauck/Noftz, EU-Sozialrecht, Art. 3 VO 883/2004, Rn. 49.

46 Anhang Ziff. 4 lit. a) VO (EG) Nr. 629/2006, ABl. L 114 vom 27.4.2006, S. 4.

47 LSG Bayern InfAuslR 2009, S. 260, 262; H. Mangold/K. Pattar, VSSR 2008, S. 243, 253.

48 E. Eichenhofer in Fuchs, Europäisches Sozialrecht (5. Auflage), Art. 4 VO (EG) 883/2004, Rn. 2 und 12; A. Otting in Hauck/Noftz, EU-Sozialrecht, Art. 4 VO 883/2004, Rn. 7. 
Im Hinblick auf die Staatsangehörigkeit der Empfänger von Leistungen sozialer Sicherheit begünstigt die Koordinierung nicht nur Unionsbürger. Das Gleichbehandlungsgebot erstreckt sich auf alle „Personen, für die diese Verordnung gilt“. Der persönliche Geltungsbereich ist in Art. 2 VO (EG) 883/2004 umschrieben und umfasst neben den Unionsbürgern Staatenlose und Flüchtlinge sowie deren Familienangehörige und Hinterbliebene. Andere Drittstaatsangehörige sind aufgrund Art. 1 VO (EU) $1231 / 2010$ in den Geltungsbereich des koordinierenden Sozialrechts einbezogen, sofern sie sich rechtmäßig in einem Mitgliedstaat aufhalten und ein grenzüberschreitender Sachverhalt vorliegt. Damit gilt das Gebot der Inländergleichbehandlung auch für sie, so dass sich ihr Anspruch auf Grundsicherung für Arbeitsuchende ebenfalls von Beginn ihres Aufenthalts an ausschließlich nach § 7 I 1 SGB II richten kann.

\section{(3) Statutenwechsel als Anknüpfungsmoment}

Die Regelungen des koordinierenden Sozialrechts zur Bestimmung des zuständigen Staats spiegeln die Anknüpfung an einen Statutenwechsel wider. Nach Art. 11 II VO (EG) 883/224 unterliegt eine Person, die aufgrund oder infolge einer Beschäftigung eine Geldleistung bezieht, weiterhin dem Recht des Beschäftigungsstaats. Dies umfasst auch Arbeitslose. ${ }^{49}$ Nichterwerbstätige, die keine solchen Leistungen beziehen - etwa weil sie die notwendigen Beitragszeiten nicht erfüllt oder noch nie eine Beschäftigung ausgeübt haben - unterliegen demgegenüber nach Art. 11 III lit. e) VO (EG) 883/2004 dem Recht des Wohnstaats, i.e. der Staat des gewöhnlichen Aufenthalts.

Die Regelungen zur Koordinierung der Leistungen bei Arbeitslosigkeit stellen sicher, dass Erwerbslose auch im Falle der Arbeitssuche in anderen Mitgliedstaaten finanziell abgesichert sind. Art. 64 VO (EG) 883/2004 belässt die Zuständigkeit für die Erbringung von Leistungen bei Arbeitslosigkeit im Regelfall für drei Monate beim Träger des Herkunftslands. Diese Leistungen sind als Einkommen i.S.v. § 11 SGB II zu bewerten, sodass ihr Bezug die Hilfebedürftigkeit i.S.v. $§ § 7$ I Nr. 2, 9 SGB II entfallen lassen, zumindest aber mindern kann. Auch an dieser Stelle wird deutlich, dass das europäische Recht den Übergang der Verantwortung eines Staats für die soziale Sicherung Erwerbsloser auf einen anderen an einen Statutenwechsel knüpft. Erst wenn ein Arbeitsuchender nicht rechtzeitig innerhalb der Dreimonatsfrist in sein Herkunftsland zurückkehrt, verliert er endgültig seine Ansprüche gegen den dortigen Träger. ${ }^{50}$ Bezieht ein Arbeitsuchender keine Leistungen vom Träger des Beschäftigungsstaats, begründet aber seinen Wohnsitz in einem anderen Mitgliedstaat - auch um dort Arbeit zu suchen - ist ihm der Zugang zu den Leistungen sozialer Sicherheit nach den dort geltenden Regelungen eröffnet. Solange also die Aufenthaltsbegründung und -fortführung vom Aufenthaltsstaat nicht infrage gestellt werden, tritt ein Statutenwechsel ein, der eine Leistungsberechtigung auch für Leistungen an Arbeitsuchende eröffnet.

49 S. Devetzi in Hauck/Noftz/Eichenhofer, EU-Sozialrecht, Art. 11, Rn. 20.

50 R. Schlegel in Hauck/Noftz, EU-Sozialrecht, Art. 64 VO 883/2004, Rn. 35. 


\section{Völkerrechtlich begründete Gleichbehandlungsansprüche}

Gewährt das Europarecht subjektive Rechte auf Gleichbehandlung regelmäßig nur in grenzüberschreitenden Konstellationen, sind völkerrechtliche Gewährleistungen nicht von einem Grenzübertritt abhängig. Als Menschenrechte knüpfen sie allein an das Mensch-Sein an und vermögen daher einen weiter gefassten Zugang zur gleichberechtigten Gewährung von Leistungen sozialer Sicherheit zu eröffnen.

a) Differenzierungen nach der Staatsangehörigkeit im Lichte des Europäischen Fürsorgeabkommens

Das Europäische Fürsorgeabkommen (EFA) - ein vom Europarat vermitteltes multilaterales Abkommen - garantiert die Gleichbehandlung der Angehörigen der Vertragsstaaten in den Leistungen der sozialen und der Gesundheitsfürsorge. Das Abkommen präzisiert den in Art. 13 ESC verankerten Grundsatz, dass jeder Mittellose ungeachtet seiner Staatsangehörigkeit Anspruch auf die Fürsorgeleistungen des Aufenthaltsstaats hat.

\section{(1) Leistungen der Grundsicherung für Arbeitsuchende als Fürsorgeleistungen}

Der sachliche Anwendungsbereich des EFA erstreckt sich auf Leistungen der Fürsorge. Darunter sind nach Art. 2 a) lit. i) EFA die Leistungen zu verstehen, die an Personen ohne ausreichende eigene Mittel gewährt werden, damit diese ihren Lebensbedarf decken können. Die Normen, auf die das EFA anzuwenden ist, sind im Anhang I des Abkommens aufgeführt, Art. 2 b) EFA. Die Leistungen der Grundsicherung sind zwar dort nicht explizit aufgeführt. Die Anhänge zum EFA haben jedoch rein deklaratorischen Charakter. Würde man das Abkommen allein auf die in die Anhänge aufgenommenen Leistungen beschränken, wäre der Gehalt des Gleichbehandlungsanspruchs aus Art. 1 EFA in das Belieben der Vertragsstaaten gestellt, die auf diese Weise anderen als den eigenen Staatsangehörigen bestimmte Fürsorgeleistungen vorenthalten könnten. Die Aufführung eines Gesetzes ist daher nicht konstitutiv für dessen Bewertung als Fürsorgeleistung. ${ }^{51}$

\section{(2) Persönlicher Anwendungsbereich des Gleichbehandlungsanspruchs}

Gemäß Art. 1 EFA sind die Angehörigen der vertragsschließenden Staaten berechtigt, in anderen Konventionsstaaten Fürsorgeleistungen unter den gleichen Bedingungen wie Inländer in Anspruch zu nehmen.

Voraussetzung der Inländergleichbehandlung ist der erlaubte Aufenthalt. Der Aufenthalt gilt nach Art. 11 lit. a) EFA so lange als erlaubt, wie der fremde Staatsangehörige in Besitz einer Erlaubnis ist, die ihm den Aufenthalt in diesem Staat gestattet. Die Erlaubnis fehlt dagegen, sobald eine wirksame und vollstreckbare Anordnung zum Ver-

51 A. Brühl/D. Schoch in Münder, LPK-SGB II, § 7, Rn. 35; H. Mangold/K. Pattar, VSSR 2008, S. 243, 261; BVerwGE 111, 200, 206; BSG, Urt. v. 19.10.2010 (B 14 AS 23/10 R), Rn. 34juris; LSG Niedersachsen-Bremen, Beschl v. 14.1.2008 (L 8 SO 88/07 ER), Rn. 40-juris. 
lassen des Landes besteht, Art. 11 lit. b) EFA. Der aufenthaltsrechtliche Rahmen für die Teilhabeansprüche nach dem EFA ist damit deckungsgleich mit dem des Unionsrechts, nachdem ein Aufenthalt auch ohne formellen Titel so lange als erlaubt gilt, bis der Aufenthaltsstaat dessen Beendigung verfügt hat.

Auf das EFA kann sich jedoch nicht berufen, wer sich missbräuchlich verhält und sich allein zu dem Zweck in einen Vertragsstaat begibt, um dort Fürsorgeleistungen in Anspruch zu nehmen. ${ }^{52}$ Namentlich wird vertreten, dass der Anwendungsbereich des Abkommens nicht eröffnet sei, wenn die Bedürftigkeit bereits bei der Einreise bestand, was regelmäßig auf Personen, die allein zum Zwecke der Arbeitssuche einreisen, zutreffen dürfte. Zwar enthalte das EFA keine entsprechende Öffnungsklausel, jedoch könne es den ratifizierenden Staaten nicht verwehrt sein, die missbräuchliche Inanspruchnahme von Fürsorgeleistungen zu verhindern. ${ }^{53}$ Nach dieser Auffassung wäre der persönliche Anwendungsbereich des Gleichbehandlungsanspruchs insoweit zu reduzieren, dass bedürftige Einreisende von ihm nicht erfasst seien. Dies überzeugt jedoch nicht, bietet doch das Abstellen auf den erlaubten Aufenthalt in Art. 1 EFA hinreichende Gewähr für eine Missbrauchskontrolle. Für die Angehörigen der EU-Mitgliedstaaten, die das EFA ratifiziert haben, gilt eine Vermutung der Freizügigkeit, die es durch einen nationalen Rechtsakt zu entkräften gilt. Die Angehörigen der übrigen Vertragsstaaten des EFA benötigen für Einreise und Aufenthalt in die Bundesrepublik ohnehin eines Aufenthaltstitels, der nach $\S 5$ I Nr. 1 AufenthG unter anderem an den Nachweis hinreichender Existenzmittel geknüpft ist. Eine generelle Ausnahme von der Inländergleichbehandlung ist daher nicht angezeigt. ${ }^{54}$

\section{b) Sonderregelungen für Ausländer im Lichte der EMRK}

Art. 14 EMRK statuiert ein Diskriminierungsverbot u.a. im Hinblick auf die „Rasse“ sowie wegen der nationalen oder sozialen Herkunft, der Zugehörigkeit zu einer nationalen Minderheit oder eines sonstigen Status. Die Staatsangehörigkeit wird zwar nicht explizit erwähnt, ist aber durch die sonstigen Merkmale, insbesondere das Verbot der Rassendiskriminierung bzw. das Verbot der Diskriminierung aufgrund der nationalen Herkunft erfasst. ${ }^{55}$

\section{(1) Diskriminierungsverbot des Art. 14 EMRK}

Art. 14 EMRK knüpft an die sonstigen in der EMRK und den dazu ergangenen Zusatzprotokollen verankerten Rechte an, weist also akzessorischen Charakter auf. Sein Anwendungsbereich ist eröffnet, wenn in eines der Rechte eingegriffen wird oder wenn eine Personengruppe bei der Ausübung dieser Rechte gegenüber anderen Personen be-

52 OVG Hamburg NVwZ-RR 1990, 141, 142; OVG Berlin FEVS 55, 186, 189 f.; H. Fenge, ZfSH 1979, S. 257, 266; V. Berger, Die Sozialhilfeansprüche von Ausländerinnen und Ausländern, S. 100.

53 OVG Berlin, FEVS S. 55, 186.

54 So im Ergebnis auch BSG, Urt. v. 19.10.2010 (B 14 AS 23/10 R), Rn. 40-juris.

55 J. Meyer-Ladewig, EMRK, Art. 14, Rn. 29. 
nachteiligt wird. ${ }^{56}$ Das Diskriminierungsverbot ist verletzt, wenn für die Ungleichbehandlung kein sachlicher Rechtfertigungsgrund besteht, d.h. wenn diese „,kein berechtigtes Ziel verfolgt oder wenn kein angemessenes Verhältnis zwischen den angewendeten Mitteln und dem verfolgten Ziel besteht". 57

Den Staaten ist zwar grundsätzlich ein Ermessenspielraum eingeräumt, inwieweit sie die unterschiedliche Behandlung von Sachverhalten als gerechtfertigt erachten. Insbesondere in der Wirtschafts- und Sozialpolitik ist dieser Spielraum sehr weit zu fassen, haben die nationalen Gesetzgeber und Behörden aufgrund ihrer Nähe zur inländischen Gesellschaft und den dortigen Lebensbedingungen doch eine stärkere Kompetenz zur Bewertung der diesbezüglich bestehenden öffentlichen Interessen. ${ }^{58}$ Im Gegenzug misst der Europäische Gerichtshof für Menschenrechte (EGMR) einzelnen verpönten Differenzierungsmerkmalen eine so erhebliche Bedeutung zu, dass allein an diese anknüpfende Ungleichbehandlungen der weitgehenden Entscheidungsfreiheit der Staaten entzogen sind. Dies gilt namentlich für den Rekurs auf die nationale oder die ethnische Herkunft. Eine darauf gestützte Ungleichbehandlung ist mit den Grundsätzen demokratischer Gesellschaften schlechterdings unvereinbar. ${ }^{59}$

\section{(2) Soziale Sicherheit als Eigentumsrecht}

Zwar finden sich in der EMRK keine Rechtsverbürgungen mit spezifisch sozialrechtlichem Charakter. Der EGMR hat jedoch aus Art. 1 EMRK-ZP, dem Schutz des Eigentums, umfassende Gleichbehandlungsansprüche im Recht der sozialen Sicherheit hergeleitet. In der Rechtssache Gaygusuz hat das Gericht Leistungen, auf die durch Beitragszahlungen Anwartschaften erworben wurden, dem Eigentumsschutz zugeordnet. Diese Betrachtung entspricht auch der deutschen verfassungsrechtlichen Tradition, Anwartschaften als vermögenswerte Rechtspositionen unter den Schutz von Art. 14 I GG zu stellen. ${ }^{60}$ Sehr viel weiter ging der EGMR in der Rechtssache Poirrez. Danach sind auch solche Leistungen der Eigentumsgarantie des Art. 1 EMRK-ZP unterworfen, die nicht auf Beitragszahlungen beruhen. Die Begründung stützt sich darauf, dass der Kläger über einen rechtmäßigen Aufenthalt verfügte und bereits Leistungen der Sozialhilfe bezog. Mit Ausnahme der Staatsangehörigkeit hatte er alle Voraussetzungen für den zusätzlichen Bezug einer Behindertenbeihilfe erfüllt, sodass mangels jedweder objektiver Rechtfertigung des Staatsangehörigkeitserfordernisses das Gleichbehandlungsgebot aus Art. 14 EMRK verletzt sei.

Dieses Urteil führt der EGMR in seiner Rechtsprechung zum Eigentumsschutz konsequent fort. Er gewährt zwar kein allgemeines Recht zum Erwerb von Eigentum. Von der Garantie des Art. 1 EMRK-ZP sind jedoch alle Forderungen und Ansprüche auf Leistungen vermögensrechtlicher Natur erfasst, auf die die berechtigte Erwartung ihrer

56 EGMR NVwZ 2006, S. 917, 917 (Okpisz); J. Meyer-Ladewig, EMRK, Art. 14, Rn. 5 f.; S. Schmahl/T. Winkler, AVR 48 (2010), S. 405, 418.

57 EGMR NJW 2002, S. 2851, 2855 (Pretty); NVwZ 2008, S. 533, 534 (D.H.).

58 EGMR, Urt. v. 21.2.1986, Az. 8793/79, Rn. 46 (James); Urt. v. 23.10.1997, Az. 117/1996/736/933-935, Rn. 80 ff. (National and Provincial Building Society); Urt. v. 27.11.2007, Az. 77782/01, Rn. 48 (Luczek); NJW-RR 2009, S. 1606, 1609 (Burden).

59 EGMR InfAuslR 1997, 1 (2) (Gaygusuz); EGMR ZESAR 2004, S. 142, 143 (Poirrez).

60 BVerfGE 72, 9; dazu ausführlich E. Eichenhofer, Sozialrecht, Rn. 133. 
Realisierung besteht. Sobald die im nationalen Recht niedergelegten Anspruchsvoraussetzungen erfüllt sind, entfaltet der menschenrechtliche Eigentumsschutz seine Wirkung. Dies gilt auch für Sozialleistungen, ohne dass es auf deren Finanzierung durch Beiträge oder aus sonstigen Quellen ankommt. ${ }^{61}$ Mit dieser Rechtsprechung hat der EGMR keineswegs einen uneingeschränkten Zugang zu allen Sozialleistungen für jedermann eröffnet, sondern dem nationalen Gesetzgeber stets weiten Gestaltungsspielraum eingeräumt. Ansprüche auf Leistungen sozialer Sicherheit können daher auch im Interesse der fiskalischen Konsolidierung von bestimmten Voraussetzungen abhängig gemacht werden. Eine Verletzung der menschenrechtlichen Garantien der EMRK kommt erst in Betracht, wenn ein Antragsteller abgesehen von der Staatsangehörigkeit des Aufenthaltsstaats alle sonstigen Bedingungen und Leistungsvoraussetzungen erfüllt. ${ }^{62}$

Ausländern ist es prinzipiell möglich, die in $\S 7$ I 1 SGB II verankerten Anspruchsvoraussetzungen zu erfüllen. Die in § 7 I 2 SGB II niedergelegte Differenzierung zwischen Deutschen und Ausländern ist mit Blick auf den menschenrechtlichen Gehalt der Art. 14 EMRK, 1 EMRK-ZP nicht gerechtfertigt. Der Rekurs auf eine besondere Verantwortung für die eigenen Staatsangehörigen ist nach Auffassung des EGMR unzulässig. ${ }^{63}$ Existenzsichernde Leistungen können nicht an besondere personelle Bindungen, wie sie durch den Erwerb einer Staatsangehörigkeit begründet oder verfestigt werden, anknüpfen. Sie sind als Recht aus dem bloßen Mensch-Sein heraus zu gewähren, würde anderenfalls das Sozialrecht der menschenrechtlichen Prüfung entzogen bleiben. Auf den Gleichbehandlungsanspruch im Grundsicherungsrecht können sich daher im Einklang mit Art. 1 EMRK alle Personen ungeachtet ihrer Zugehörigkeit zu einem Konventionsstaat berufen, die der Hoheitsgewalt des deutschen Sozialstaats unterworfen sind.

\section{Fazit}

Nach dem Willen des Gesetzgebers ist der Zugang zu den Leistungen der Grundsicherung für Arbeitsuchende dem Grunde nach nur Deutschen sowie Ausländern eröffnet, die über hinreichende Mittel zum Lebensunterhalt sowie erworbene Rentenanwartschaften verfügen, dass eine Belastung des Sozialstaats von vornherein nahezu ausgeschlossen ist. Die Differenzierung wird damit begründet, dass die Leistungen der Sozialhilfe eine Eingliederung in die inländische Gesellschaft bewirken sollen. Diese sei im Grunde nur Deutschen geschuldet, da nur sie per se ein uneingeschränktes Aufenthaltsrecht in der Bundesrepublik innehaben.

Eine solche Differenzierung wird jedoch weder vom Europa- noch vom Völkerrecht gedeckt. Zwar eröffnet das supranationale Recht keine kosmopolitische Freizügigkeit. Es vermittelt aber all jenen Ansprüche auf Fürsorgeleistungen, die sich rechtmäßig in einem Staat aufhalten. Wesentliches Motiv der Leistungen nach dem SGB II ist die Sicherung einer menschenwürdigen Existenz. Die Achtung der Menschenwürde obliegt aller staatlichen Gewalt gegenüber jedermann, ungeachtet seiner Nationalität oder Her-

61 S. Schmahl/T. Winkler, AVR 48 (2010), S. 405, 424.

62 U. Davy, ZIAS 2000, S. 221, 240; K. Hailbronner, JZ 1997, S. 397, 400.

63 EGMR InfAusIR 1997, 1 (3) (Gaygusuz); dazu ausführlich U. Davy, ZIAS 2000, S. 221, 238 f. 
kunft, Art. 1 I, III GG. Wenn die sozialen Hilfen ein menschenwürdiges Dasein ermöglichen sollen, stellt sich angesichts der Unveräußerlichkeit der Menschenwürde nach Art. 1 I GG die Frage, ob Differenzierungen im Leistungsumfang überhaupt zulässig sind. Haben Menschen unterschiedlicher Herkunft oder mit unterschiedlichem Aufenthaltsstatus eine voneinander verschiedene Menschenwürde? Dies wäre jedoch ein Fehlschluss. Die Menschenwürde steht jedermann universell und überall zu. Sie zu schützen und zu sichern, ist eine Aufgabe jedweder öffentlicher Gewalt. Die Differenzierungen erklären sich allein aus der Frage der „Zuständigkeit“ eines Sozialstaats für die Gewährleistung eines menschenwürdigen Daseins. Sind die Bindungen an den Aufenthaltsstaat noch nicht so verfestigt, dass die Rückreise in das Herkunftsland nicht schlechterdings unzumutbar wäre, besteht die Verpflichtung zur sozialen Fürsorge nur eingeschränkt. Durch das Erfordernis des gewöhnlichen Aufenthalts in $\S 7$ I 1 Nr. 4 SGB II ist bereits hinreichend Vorsorge zur Verhinderung einer Sozialleistungsmigration getroffen. Besteht die sozialrechtliche Verantwortlichkeit des Herkunftslandes fort und kann diese auch in zumutbarer Weise in Anspruch genommen werden - was regelmäßig nur bei den Deutschen ohnehin gleichgestellten Flüchtlingen und Asylberechtigten nicht der Fall ist -, ist dieser im Erfordernis eines zukunftsoffenen Aufenthalts gründende Leistungsausschluss unproblematisch. Weder ist das aus der Menschenwürdegarantie nach Art. 1 I GG i.V.m. dem Sozialstaatsprinzip nach Art. 20 I GG resultierende Grundrecht auf Sicherung einer menschenwürdigen Existenz verletzt. Denn wer Fürsorgeleistungen in seinem Herkunftsstaat beanspruchen kann, wird durch $\S 7$ I 1 Nr. 4 SGB II nicht jedweder existenzsichernder Leistungen beraubt. Noch ist der Gleichbehandlungsgrundsatz aus Art. 3 I GG tangiert, stellt doch der nicht erfolgte Statutenwechsel einen hinreichend tragfähigen Grund für die Verweigerung der Grundsicherungsleistungen dar.

Ist eine Person jedoch in die Aufenthaltsgesellschaft integriert, weil ihr Aufenthalt geduldet oder sogar rechtlich verfestigt ist, ist sie umfassend gegen soziale Exklusion zu sichern. Sie ist dann Teilhaber der Solidargemeinschaft. Ihr Fürsorge zu gewähren, sichert nicht zuletzt den sozialen Frieden im Aufenthaltsstaat. ${ }^{64}$

64 C. Schönberger, Unionsbürger, S. 364. 